diagnosed intracranial tumor by pneumoventriculogram. By operation right falx fibroblastic meningioma was removed totally. She is now 2 years and 9 months old, normally developed mentally and physically.

Case 2: 7 months old male developed vomiting and enlarged head and found bilateral optic nerve atrophy. By ventricle tap brownish color mucus was obtained and PVG showed upper displacement of the lateral ventricles. Surgery showed tratoma on the midline including the $3 \mathrm{rd}$ ventricle. He suddenly died one year after surgery.

Case 3: 10 months old female became leathergic, spastic paresis in extremities and the size of the head increased. PVG showed shift of lateral ventricles. Operation revealed large left intracerebral cyst (100 c.c.) with undifferentiated glioma. Postoperative irradiation of cobalt $4000 \mathrm{r}$. was given. She is now 3 years and 7 months old having residual spastic paresis and moderate mentall retardation.

Case 4: 11 months old male had occasional fever and vomiting, his head enlarged. PVG showed hydrocephalus and protein in ventricle fluid was 420 $\mathrm{mgm} / \mathrm{dl}$. Ventriculo-pleura shunt was done however, died in 2 months after progressive deterioration. Autopsy revealed spongioblastoma diffusely invaded the base of brain and mid-brain.

Domestic and foreign literatures were reviewed, 68 cases of intracranial tumors reported in the age below 12 months. Supra-tentorial tumors were 3.4 times more than infra-tentorial ones. Most frequent was teratoma 17 cases then ependymoma 10, choroidal plexus papilloma 8 and medulloblastoma 6 .

Presented our case I can be the youngest, successful, totally removed meningioma with the case reported by J.N. Taptas. These 4 cases are all first babies and delivered from the fairly advanced age mothers 40, 26, 27, and 32 years old and the symptoms i.e., vomiting, change of mood and enlargement of the head appeared within the short period.

\title{
i-19. Clinical Survey of 6 Cases of Hydroencephalodysplasia
}

\author{
Susumu IshiKawa, Katsuaki Sakoda, Yasuo Takeichi, Hideto NishiKaWa, \\ Katsumasa Nakano, Kenji Tomihara and Hiroshi Kumano \\ 2nd Department of Surgery, Hiroshima University School of Medicine
}

During the past 6 years we have identified 6 cases of hydroencephalodysplasia. The ages of the patients ranged from 3 months to 2 years; 4 of the patients were males, 2 females. In the history of one was a premature delivery at the 8 month of pregnancy, in the remaining 5 there were no abnormal conditions in pregnancy and delivery. The increase in size of head was seen in 5 , and 2 of whom demonstrated transillumination of the skull. One patient was 
noticed as having scoliosis and craniostenosis with a head circumference of $38 \mathrm{~cm}$ at 3 months of age (turricephaly). Convulsions, either generalized or Jacksonian, were noticed in 4 . Other neurological findings were visual disturbance, muscular rigidity, hyperreflexia and psychomotor arrest. But 2 patients were neurologically asymptomatic except for enlarged head in spite of the large defect in the right cerebral hemisphere. Funduscopic studies showed optic atrophy in 4. EEG showed flatty pattern corresponding to cavity formation and diffuse slowing. Epileptic factors were detected only in one case. Ventriculography revealed abnormal air collections and angiography presented avascular areas and shift of arteries. An exploratory craniotomy was performed in some cases. Immediately beneath the dura a veil-like membrane enclosed an enormous fluid-filled cavity, which was separated by some septa. Cystoatrial shunt was done in 2 cases; cystoperitoneal shunt in 2 . Osteotomy and plexectomy were performed in the case of craniostenosis. No operation was done in one. Of these, the case of craniostenosis had no subsequent follow-up. 2 patients died of infection and obstruction of the shunt tube after cystoperitoneal shunt and cystoatrial shunt respectively. Another 3 are alive and fairly well.

\title{
i-20. The Experience of Ventriculo-atrial Shunt Operation for Hydrocephalus
}

\author{
Shinken Kuramoto, Shigeyuke Takaki, Yoshiomi TakamiYa and \\ Ryoichi ONAKA \\ Neurosurgical Clinic, Kurume University School of Medicine
}

The ventriculo-atrial shunt operation is generally operated at present as the method of the best result in the treatment of hydrocephalus.

We experienced 19 cases of hydrocephalus: The ventriculo-atrial shunt operation was operated for 16 cases. We used the homemade tube for 5 cases and Spitz-Holter valve for 11 cases.

The complications of ventriculo-atrial shunt operation are mainly the occlusion and infection.

We had 7 cases of occlusion and took the revision at ten times. The protein in spinal fluide indicated almost high concentration in the case of occlusion.

About infection, in the case of using the homemade tube, so many patients died by sepsis during one or three months post operation. But in the case of using Holter valve we have not experienced yet such cases above mentioned.

In order to get the good result in the treatment of hydrocephalus, we consider it is the most necessary introduce certainly the tube between the ventricle and atrium and then to follow up enough postoperative supervision. 